\title{
LETTER
}

doi:10.1017/\$1041610216000673

\section{Preserved brain metabolic activity at the age of 96 years}

Loss of brain tissue becomes notable to cerebral magnetic resonance imaging (MRI) at age 30 years, and progresses more rapidly from mid 60s. The incidence of dementia increases exponentially with age, and is all too frequent in the oldest old ( $\geq 90$ years of age), the fastest growing age group in many countries. However, brain pathology and cognitive decline are not inevitable, even at extremely old age (den Dunnen et al., 2008).

Therefore, considerable effort is currently directed toward developing interventions to ameliorate aging processes, such that people might live longer without developing severe disabilities. One approach to understand successful aging is to study very old healthy subjects. Functional brain imaging in the oldest old might contribute to improved understanding of the mechanisms underlying successful aging of the brain. However, till now there have been no imaging studies of brain metabolism specifically in the oldest old.

Against this background, we present the case of a 96 years old woman with a completely preserved pattern of brain metabolic activity as measured by positron-emission tomography (PET) with the glucose analogue $18 \mathrm{~F}$ fluorodeoxyglucose (FDG) (Figure 1). Brain FDG PET is a well-established functional imaging modality for non-invasive in vivo assessment of brain metabolic activity, which is generally considered as surrogate marker of signalling-related synaptic activity. Brain FDG PET is widely used both in research and routine clinical patient care. Synapse loss resulting in reduced FDG uptake, particularly in the posterior cingulate cortex/precuneus area as well as in parietotemporal association cortices, is the major correlate of cognitive impairment in Alzheimer's disease ( $\mathrm{AD}$, Figure 1), not only in the dementia phase but already in the phase of mild cognitive impairment. Reduction of FDG uptake associated with healthy aging is most pronounced in frontal and parietal cortex (Knopman et al., 2014).

The woman presented here participates in an ongoing prospective clinical study on the utility of brain FDG PET for the etiological diagnosis of clinically uncertain cognitive impairment in geriatric inpatients (WHO Trials Registry DRKS00005041). Study procedures included standardized patient history, physical/neurological examination, blood/urine laboratory tests, detailed neuropsychological testing, MRI of the brain, FDG PET of the brain, and ApoE genotyping. PET images were processed as described previously (Lange et al., 2015). Voxel-by-voxel and regionof-interest (ROI)-based statistical testing was performed using the statistical parametric mapping software package (SPM version 8) and a normal database of 32 healthy control participants aged $73.8 \pm 4.6$ years $(22$ females, MMSE $28.9 \pm 1.2$ ). Detailed neuropsychological testing was repeated after 16 months.

The women had been hospitalized in a geriatrics ward for acute care because of recurrent diarrhea and general cachexia. In her younger years, she had been an opera singer and music teacher (in total 45 years), with 15 years of education. She had not experienced memory problems or restrictions in her daily activities prior to hospitalization. There was no family history of dementia or cardiovascular diseases. Her medical history included lactose and fructose intolerance, mild hypertension, as well as chronic renal insufficiency. Neurological examination was quite unremarkable. Laboratory examination showed a mild protein deficiency, increased renal retention parameters, normocytic anaemia and B- and D-hypovitaminosis. Under treatment, her general condition recovered rapidly. However, improvement of cognition clearly lagged behind (MMSE score 23 of 30 points), which raised suspicion of first manifestations of $\mathrm{AD}$, such that she was enrolled in the clinical study.

Detailed neuropsychological testing performed a few days after inclusion in the study revealed quite normal performance in all domains and no signs of depressive mood (Supplementary Table). Delirium at this time point was excluded by the Nursing Delirium Score and the Delirium Rating Scale. ApoE genotype was $2 / 3$. Brain MRI was free of major vascular pathology (no large vessel stroke, no recent small subcortical infarcts, multiple chronic lacunes in frontal and parietal white matter, no microbleeds, no superficial siderosis, mild to moderate white matter hyperintensities with Wahlund score 9) and showed only mild cortical atrophy (Scheltens score 2 for medial temporal lobe). Brain FDG PET showed completely preserved brain metabolic activity, not only by visual inspection (Figure 1), but also by statistical testing against the group of healthy subjects: regional FDG uptake scaled to the pons was within mean \pm 2 


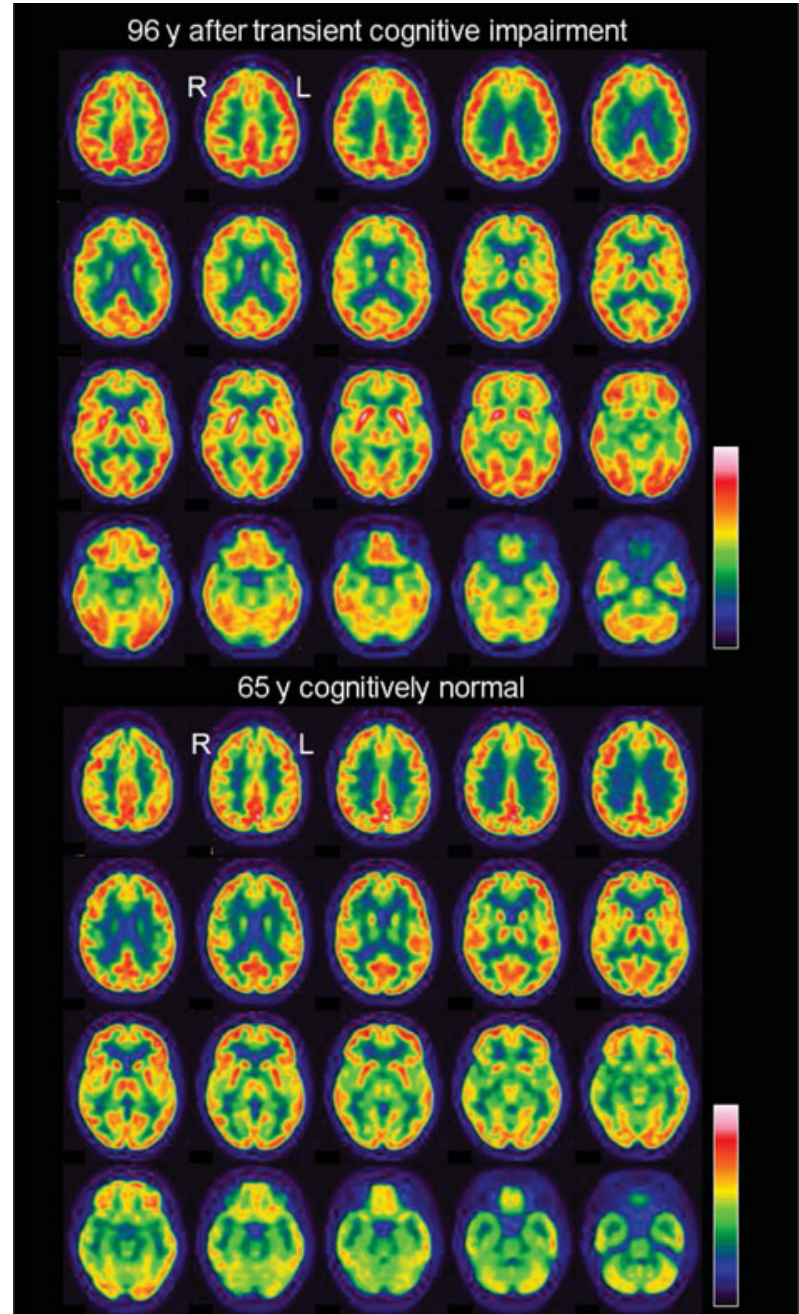

$96 \mathrm{y}$ with mild Alzheimer's type dementia

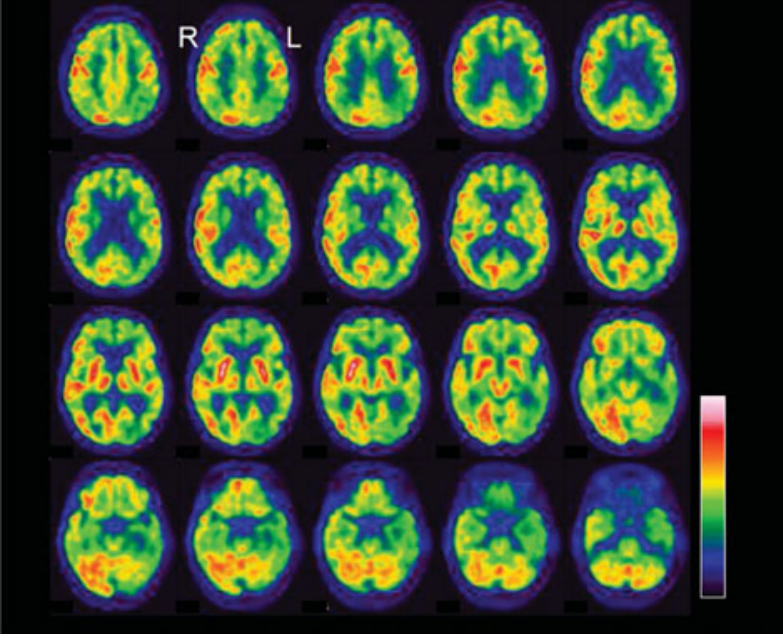

Figure 1. Completely normal brain FDG PET of the cognitively normal 96 years old woman after recovery from transient cognitive impairment associated with exsiccosis and deterioration of the general condition. Brain FDG PET of a 65 years old cognitively normal woman and of a 96 years old woman with mild dementia probably due to Alzheimer's disease are shown for comparison. standard deviations of the controls in all ROIs (in total 24 ROIs covering the whole brain). It was concluded that the woman's cognitive impairment that had led to inclusion in the study was a transient phenomenon due to exsiccosis and deterioration of the general condition, as is frequently observed in elderly participants (Inouye et al., 1993).

At clinical follow-up 16 months later, now at age 97 years, the woman's performance in activities of daily living and neuropsychological testing was still preserved (Supplementary Table). She ran her household alone, drove a car, and managed her financial matters by herself (e.g. online banking). She was still engaged with her hobbies like playing the piano and playing chess, reading, visiting the theatre, and writing her autobiography on a personal computer.

Healthy brain aging is poorly documented in the extremely elderly, and our case is the very oldest person with completely normal brain FDG PET findings hitherto reported in the literature. The lack of statistically significant differences in voxel- and ROI-based single subject analysis of her FDG PET against a group of healthy controls aged $73.8 \pm 4.7$ years classifies brain metabolic activity of the 96 years old woman at the level of "normal" healthy aging about 20 years younger. This suggests that metabolic activity can be preserved throughout the whole brain until very old age.

Finally, brain FDG PET has been shown to be predictive not only of the development of Alzheimer's dementia in patients with amnestic mild cognitive impairment, but also of memory decline in healthy participants (Caselli et al., 2008). The finding of normal cognition at the 16 months follow-up in the present case suggests that normal synaptic brain activity as measured by brain FDGPET might be predictive of stable cognitive abilities during the next year also in very old persons.

\section{Conflict of interest}

Lothar Spies is employee of jung diagnostics $\mathrm{GmbH}$. There are no financial interests or any other conflicts of interests associated with this work.

\section{Funding}

This study was supported by the Regional Development Fund of the European Union (references 10153407, 10153971, 10153458, 10153460-63).

\section{Description of authors' roles}

IA and RB contributed to design or conceptualization of the study, to analysis or interpretation of the data, and to drafting or revising the manuscript 
for intellectual content. LS, JBF, AM, and CL contributed to design or conceptualization of the study, and to analysis or interpretation of the data. JS, KR, and ES-T contributed to design or conceptualization of the study, and to drafting or revising the manuscript for intellectual content.

\section{Supplementary Material}

To view supplementary material for this article, please visit http://dx.doi.org/10.1017/ S1041610216000673.

\section{References}

Caselli, R. J., Chen, K., Lee, W., Alexander, G. E. and Reiman, E. M. (2008). Correlating cerebral hypometabolism with future memory decline in subsequent converters to amnestic pre-mild cognitive impairment. Archives of Neurology, 65, 1231-1236.

den Dunnen, W. F. et al. (2008). No disease in the brain of a 115-year-old woman. Neurobiology of Aging, 29, $1127-1132$

Inouye, S. K., Viscoli, C. M., Horwitz, R. I., Hurst, L. D. and Tinetti, M. E. (1993). A predictive model for delirium in hospitalized elderly medical patients based on admission characteristics. Annals of Internal Medicine, 119, 474-481.

Knopman, D. S. et al. (2014). 18F-fluorodeoxyglucose positron emission tomography, aging, and apolipoprotein $\mathrm{E}$ genotype in cognitively normal persons. Neurobiology of Aging, 35, 2096-2106.

Lange, C., Suppa, P., Frings, L., Brenner, W., Spies, L. and Buchert, R. (2015). Optimization of statistical single subject analysis of brain FDG PET for the prognosis of mild cognitive impairment-to-Alzheimer's disease conversion. Fournal of Alzheimers Disease, 49, 945-959.

IVAyla Apostolova, ${ }^{1}$ CATHARINA LANGe, ${ }^{2}$ LOTHAR SPIES, ${ }^{3}$ KERSTIN RitTER, ${ }^{4}$ ANJA MÄURER, ${ }^{5}$ JOACHIM SEYBOLD, ${ }^{5,6}$ JOCHEN B. FiEBACH, ${ }^{7}$

Elisabeth Steinhagen-Thiessen ${ }^{8}$ AND RALPH BUCHERT ${ }^{2}$

${ }^{1}$ Department of Radiology and Nuclear Medicine, University Hospital Magdeburg, Magdeburg, Germany

${ }^{2}$ Department of Nuclear Medicine, Charité Universitätsmedizin, Berlin, Germany

${ }^{3}$ jung diagnostics $\mathrm{GmbH}$, Hamburg, Germany

${ }^{4}$ Bernstein Center for Computational Neuroscience Berlin, Charité - Universitätsmedizin, Berlin, Germany

${ }^{5}$ Evangelisches Geriatriezentrum Berlin, Berlin, Germany

${ }^{6}$ Department of Internal Medicine/Infectious Diseases and Pulmonary Medicine, Charité

-Universitätsmedizin, Berlin, Germany

${ }^{7}$ Center for Stroke Research Berlin, Charité Universitätsmedizin, Berlin, Germany ${ }^{8}$ Lipid Clinic at the Interdisciplinary Metabolism Center, Charité - Universitätsmedizin, Berlin, Germany 